\title{
A RETROSPECTIVE STUDY OF RHINO-ORBITAL MUCORMYCOSIS
} occlusion.

\section{Prof. Dr. S.} Srinivasan*

\section{Dr. P. Divya}

\section{Dr. A. Rohini}

M.S, D.O., Head Of The Department, Department Of Ophthalmology, Government Kilpauk Medical College And Hospital, Chennai- 600010. *Corresponding Author

M.S., Assitant Professor, Department Of Ophthalmology, Government Kilpauk Medical College And Hospital, Chennai-600010.

M.S.,F.I.C.O., Assitant Professor, Department Of Ophthalmology, Government Kilpauk Medical College And Hospital, Chennai-600010.

M.B.B.S., Postgraduate, M.S (OPHTHALMOLOGY), Department Of Dr. R. Jayapreethi Ophthalmology, Government Kilpauk Medical College And Hospital Chennai- 600010

BACKGROUND: Mucormycosis is a rapidly progressing, life threatening, opportunistic fungal infection caused by angioinvasive mucorales species of the phylum zygomycetes. The major risk factors of the disease are uncontrolled diabetes mellitus, COVID-19 infection,prolonged usage of high dose of systemic corticosteroids,immunocompromised status and neutropenia. These create a nidus for fungal infection through nasal mucosa which spreads up through paranasal sinuses into orbit manifesting as rhino-orbital mucormycosis.

AIIM

1. To study the epidemiology and various clinical patterns of presentation of rhino-orbital mucormycosis in post covid patients.

2. To analyse the risk factors associated with progression of rhino-orbital mucormycosis in post covid patients.

METHODS: A retrospective study of 25 patients diagnosed as rhino-orbital mucormycosis in post covid patients at Government Kilpauk Medical College Hospital between April and July 2021 was done.

RESULTS: In our study,males (64\%) are more commonly affected with age preponderance of 51-60 years(28\%). The

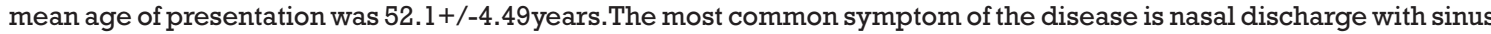
and orbital pain(52\%).The most common form of presentation is orbital apex syndrome in seven patients(28\%).Five patients had loss of vision, of which four were due to central retinal artery occlusion(16\%) and one due to cavernous sinus thrombosis.MRI imaging of these patients showed sinus involvement (100\%) in all cases ,orbital $(48 \%)$ and cerebral involvement $(8 \%)$.The major risk factors responsible for progression of disease in our study are uncontrolled diabetes, prolonged usage of high dose steroid therapy, medial wall involvement, optic nerve head involvement and artery thrombosis.

CONCLUSION: Post covid patients presenting with nasal discharge with sinus and orbital pain symptoms need to be diagnosed and managed properly to avoid spread of infection and fatal complications. Aggressive surgical debridement of infected tissues helps in increasing the survival rate of the patients. Patients with risk factors need to be closely monitored to prevent vision loss and cosmetic disfigurement surgeries.

\section{INTRODUCTION}

Mucormycosis is an angioinvasive fatal infection belonging to the phylum zygomycetes which include rhizopus, mucor, rhizomucor, cunninghamella and absidia ${ }^{(1,2)}$ They transform into aseptate hyphae which proliferates and invades the vessels resulting in thrombosis, ischemia and necrosis. The most common form is rhino-orbital-cerebral mucormycosis which extends from nasalmucosa and sinus to orbit and brain. The other forms of infection are pulmonary, cutaneous, gastrointestinal and disseminated forms.

The prevalence of mucormycosis globally varies from 0.005 to 1.7 per million population. In India, the prevalence is approximately 0.14 cases per 1000 population which is 80 times higher than developed countries $\left({ }^{3,4}\right)$.Endothelial barrier disruption, poor alveolar-capillary oxygen transmission, decreased T-lymphocytes, altered neutrophil ratio are characteristic features of COVID-19 ${ }^{(5)}$. The prevalence of co-infection among COVID-19 is $63.64 \%{ }^{(6)}$ Mucormycosis has an increasing incidence among covid patients in recent trends.

The main clinical presentations of rhino-orbital mucormycosis are nasal stuffiness, nasal discharge followed by eyelid and periorbital edema, orbital pain, ptosis, proptosis, ophthalmoplegia, sudden loss of vision. CNS symptoms include seizures,facial and cranial nerve palsy.
In mucormycosis, anterior rhinoscopy shows a black, necrotic turbinate or septum due to infarction of tissue.Orbital involvement presents as proptosis, ptosis, thrombosis of vessels and ophthalmoplegia ${ }^{(7,8)}$. Intracranial invasion occurs by way of the superior orbital fissure, cribriform plate, ophthalmic vessels, and possibly via a perineural route ${ }^{[9,10]}$ MRI has a role in diagnosing the disease at an early stage. Aggressive and prompt treatment helps in preventing fatal complications. The aim of our study is to assess the epidemiology and various clinical patterns of presentation and to analyse the risk factors associated with progression of rhinoorbital mucormycosis in post covid patients.

\section{MATERIALS AND METHODS}

Study Design: Retrospective case study

Study Participants: 25 post covid patients who presented with symptoms of mucormycosis were included in this study.

Period Of Study: April 2021-July 2021

\section{Inclusion Criteria:}

1. All post covid patients who were diagnosed as mucormycosis

\section{Exclusion Criteria:}

1.Age less than 18 years 
2.Those who are not willing to participate in study.

3. All patients who had active Covid-19 infection.

Informed consent was obtained from all the patients before including them in the study.

\section{METHODOLOGY:}

All post-covid patients who presented with nasal and orbital symptoms were examined with anterior rhinoscopy, diagnostic nasal endoscopy and biopsy by ENT surgeon. Biopsy specimen was sent for both microbiological and histopathological examination. Visual acuity assessment, anterior segment examination, exophthalmometry, fundus examination using indirect ophthalmoscope, intraocular pressure using rebound tonometry, B-scan ultrasound for posterior segment examination and neurological examinations were done. Radiological investigations like Computed Tomography and Magnetic resonance imaging of paranasal sinuses, orbit and brain were done to identify the extent of lesion.

Complete blood counts, blood sugar levels, renal function test, serumelectrolytes, liver function tests were done. Creactive protein, D-Dimer, serumferritin values were also obtained. Repeat RT-PCR was done to know the infective status of the patients. Diagnosis of mucormycosis was made based on a demonstration of blackish material on diagnostic nasal endoscopy and confirmation was done by microbiological and histopathological examination.

FESS with debridement of necrotic tissue and subsequent treatment with systemic anti-fungal therapy controlled the spread of mucor infection from extending into orbital cavity.In patients with orbital spread of infection, they were treated with retrobulbar liposomal Amphotericin to contain the infection within orbital cavity. Orbital exenteration was done in selected cases to prevent spread of infection into cranial cavity. Neurophysician and Neurosurgeon managed the patients if they had cerebral involvement.

All patients were observed for clinical recovery and further progression of disease.Visual acuity assessment, extra ocular movement assessment and fundus examination were done frequently to rule out orbital involvement and progression in high risk patients.

\section{RESULTS:}

In our study,patients with age group 51-60 years(28\%) were most commonly affected followed by $41-50$ years $(24 \%)$. The mean age of presentation was 52.1+/- 4.49years. Out of 25 patients, $16(64 \%)$ were males and $9(36 \%)$ were females showing a male preponderance.In our study, left sided lesions $(56 \%)$ were more common followed by bilateral involvement (32\%).Right sided lesions were present in $12 \%$ cases.

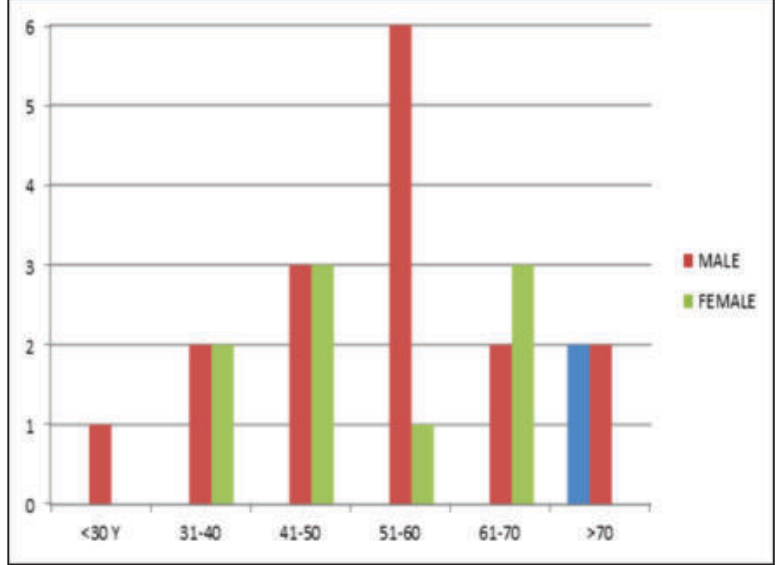

Fig-1 : BAR Graph Showing Age-gender Prevalence In Rhinoorbital Mucormycosis
The various ophthalmic symptoms in mucormycosis patients of our study is depicted in Table-1. Among these orbital pain( $52 \%)$ was the primary symptomfollowed by headache $(48 \%)$, drooping of eyelids(32\%), periorbital swelling $(28 \%)$,proptosis and restriction eye movements (24\%). $16 \%$ of patients had total loss of vision due to central retinal artery occlusion.About $44 \%$ of the patients developed nasal discharge as a main presenting symptom. The other nasal symptoms are nasal discharge,epistaxis and sinus tenderness.The primary orbital sign for most of the patients in our study are ptosis(32\%), followed by periorbital edema(28\%), proptosis(24\%) and ophthalmoplegia(24\%) (Fig-2)

Table 1:Table Depicting Ophthalmic Symptoms In Rhinoorbital Mucormycosis

\begin{tabular}{|l|l|}
\hline OPHTHALMIC SYMPTOIMS & $\begin{array}{l}\text { NO.\&PERCENTAGE OF } \\
\text { PATIENTS }\end{array}$ \\
\hline Headache & $12(48 \%)$ \\
\hline Eye pain & $13(52 \%)$ \\
\hline Drooping of eyelids & $8(32 \%)$ \\
\hline Periorbital swelling & $7(28 \%)$ \\
\hline Restriction of eye movements & $6(24 \%)$ \\
\hline Proptosis & $6(24 \%)$ \\
\hline Chemosis of conjunctiva & $5(20 \%)$ \\
\hline Loss of infraorbital sensation & $5(20 \%)$ \\
\hline Total vision loss & $5(20 \%)$ \\
\hline
\end{tabular}

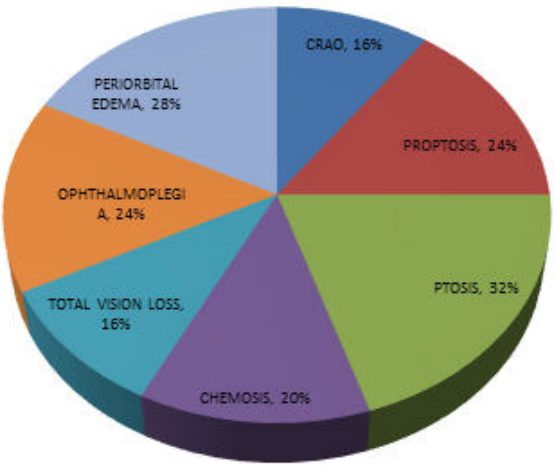

Figure-2:Primary Signs Of Rhino-orbital Mucormycosis

Various co-morbid conditions associated in mucormycosis patients of our study are depicted in Table-2. About 24 patients presented with co-morbidities in our study.

Table 2:Comorbid Conditions Associated With Rhinoorbital Mucormycosis In Our Study

\begin{tabular}{|l|l|}
\hline Comorbidities & Percentage of patients \\
\hline Diabetes mellitus & $92 \%$ \\
\hline Hypertension & $64 \%$ \\
\hline Hyperlipidemia & $8 \%$ \\
\hline Post CABG & $4 \%$ \\
\hline Ischemic Heart Disease & $4 \%$ \\
\hline Hypothyroidism & $4 \%$ \\
\hline
\end{tabular}

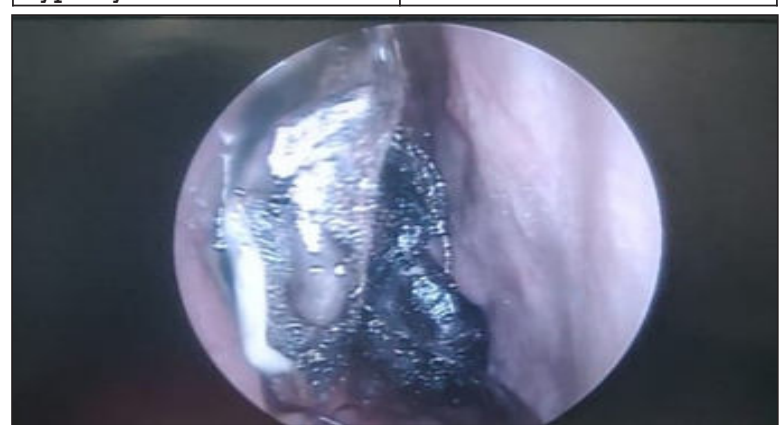

Fig-3: Diagnostic Nasal Endoscopy Showing Blackish Necrotic Material In Mucormycosis 
On anterior rhinoscopy, all patients had black bloodstained debris in the region of inferior and middle turbinate along with necrosis on nasal endoscopy(Fig-3 ).Tissue specimens for $\mathrm{KOH}$ staining and culture were obtained from all cases. Direct examination after $\mathrm{KOH}$ staining showed positive fungal filaments in 8 patients.(Fig-4).The culture was made on Sabouraud dextrose medium which showed growth of colonies in 8 patients.(Fig- 5).However in all cases, histopathological picture with hematoxylin and eosin stain showed fungal filaments of mucormycosis (Fig-6).

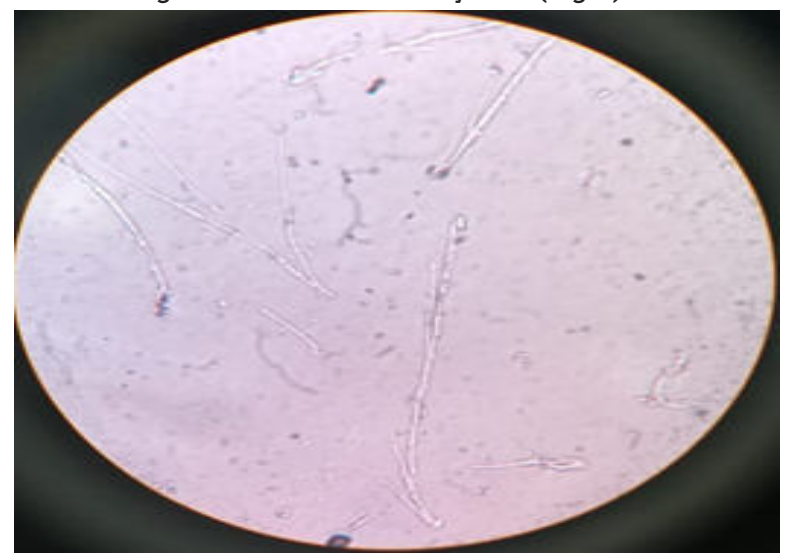

Fig-4 : $\mathrm{KOH}$ Mount Showing Fungal Filaments In Mucormycosis

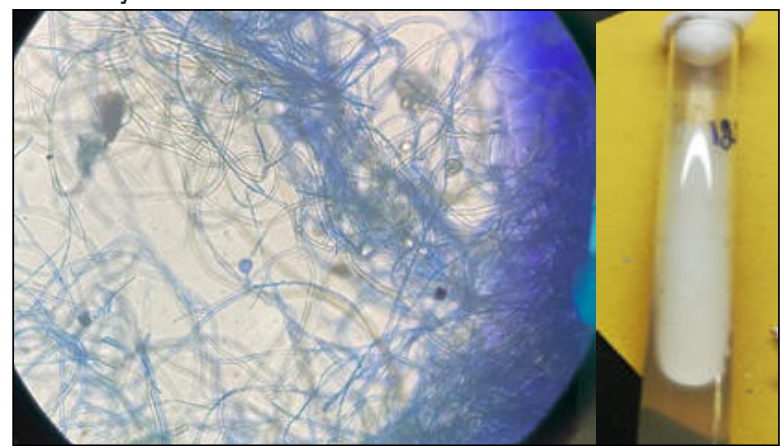

Fig-5 : Lactophenol cotton blue staining of tissue culture showing globose intact sporangium with branched sporangiophores and absent rhizoids and stolons \& Culture showing white colonies of mucor spp in sabourauds dextrose medium.

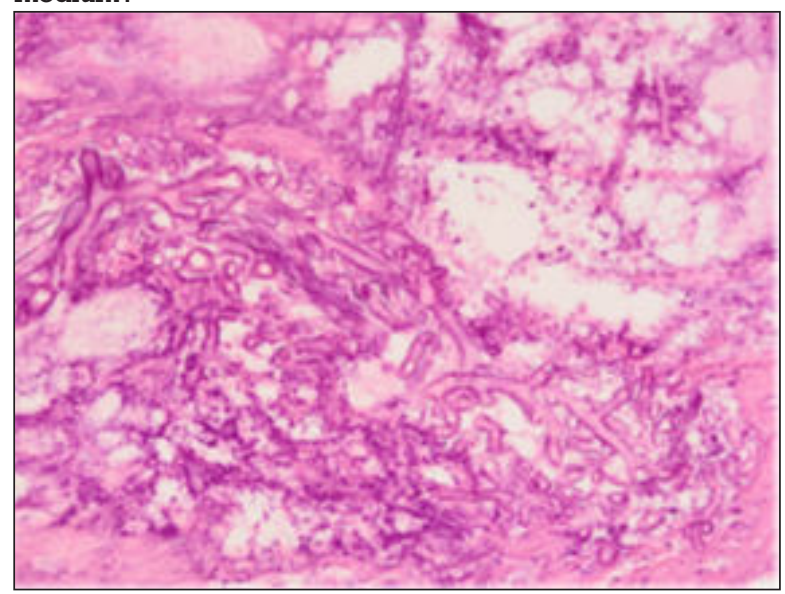

Fig-6: Histopathological Picture Showing Broad, Hyaline, Irregular Aseptate Fungal Hyphae Showing Wide Angled Branching Of Mucor SPP.

All patients underwent CT/MRI scan of paranasal sinuses, orbit and brain. Pansinusitis was found in 16 patients, and maxilla ethmoidal sinusitis was found in 9 patients (Fig-7). The thickening of sinus mucosa and extension of lesions to the orbits were observed in 12 patients(48\%) (Fig-8) (Table-3). One patient presented with thrombosis of cavernous sinuses(Fig- 9). Extension to left frontal lobe with cerebral abscess formation was noted in one patient. (Fig-12).

Table-3: MRI Findings Of Rhinorobital Mucormycosis Patients

\begin{tabular}{|l|l|l|}
\hline INVOLVEIMENT & NO.OF PATIENTS & PERCENTAGE \\
\hline SINUS/RHINO & 25 & $100 \%$ \\
\hline ORBITAL & 12 & $48 \%$ \\
\hline INTRACRANIAL & 2 & $8 \%$ \\
\hline
\end{tabular}

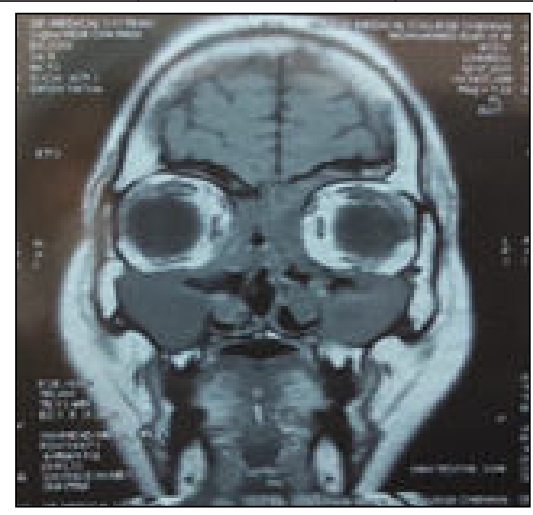

Fig-7: MRI -paranasal Sinuses Showing Hypointense Tl Lesions And Hyperintense T2 Lesions In Mucormycosis Patient

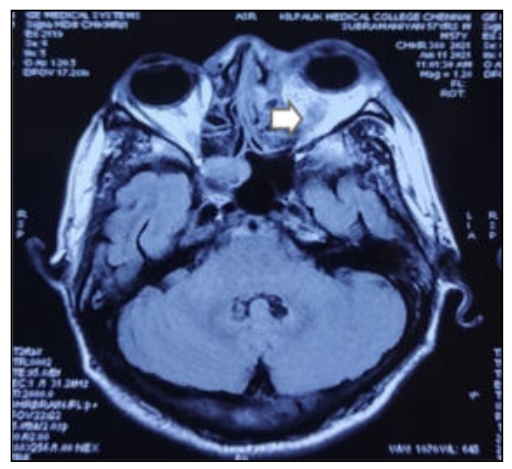

FIG-8: MRI - Orbit Showing T1 Hypointense And T2 Variable To Hyperintense Lesions In Intraconal Extraconal And Preseptal Regions Of Mucormycosis Patient

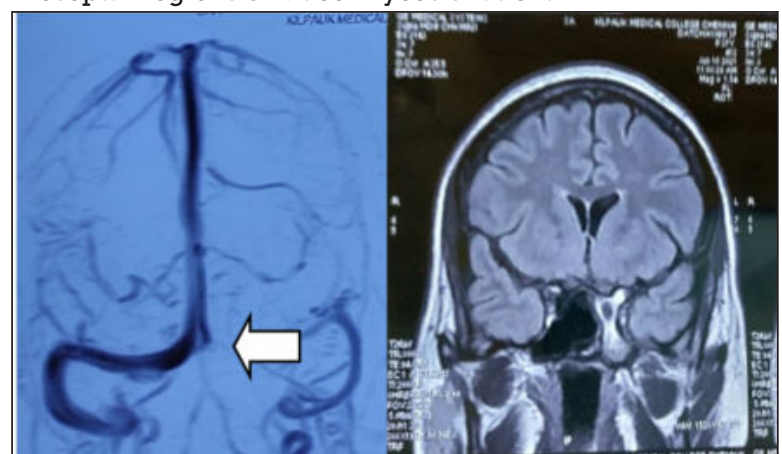

Fig-9: MRI - Brain And Orbit Showing Cavernous Sinus Thrombosis With Internal Capsule Involvement

Out of 25 patients, 14patients belong to stage-3, 9patients belong to stage- 2 and 2 patients belong to stage- 4 in our study.In our study, all cases had maxillary sinus involvement and only 12 patients had orbital extension. Among orbital presentations, 4 patients had sudden onset of painless loss of vision, proptosis,complete ptosis, chemosis, ophthalmoplegia who were diagnosed as CRAO with orbital apex syndrome (Fig-10). 2 patients presented with proptosis and 2 patients presented with ptosis and 
ophthalmoplegia(Fig-11). One patient had late presentation of cavernous sinus thrombosis with ptosis,ophthalmoplegia.

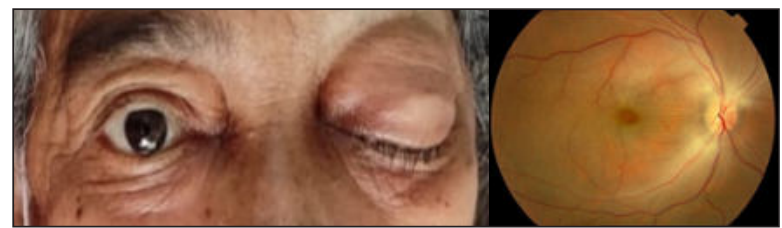

Fig-10: Profile Picture And Fundus Picture Of Patient With Orbital Apex Syndrome

In our study, all patients were treated with oral posaconazole and nasal endoscopic debridement. In patients with orbital presentation, all 12 patients were treated with retrobulbar liposomal amphotericin and systemic antifungal therapy.Two patients showed resolution of proptosis following treatment in our study (Fig-11).Among 12 patients, one patient had a progression of disease and hence treated with orbital exenteration. One patient who underwent orbital exenteration presented with cerebral extension manifesting as seizures (Fig-12).

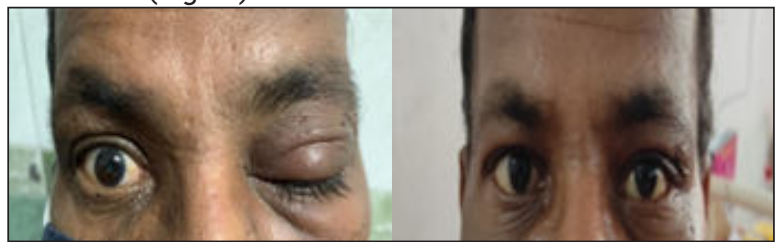

Fig-11: Patient With Left Eye Proptosis, Ptosis At The Time Of Presentation And Complete Recovery During Discharge.

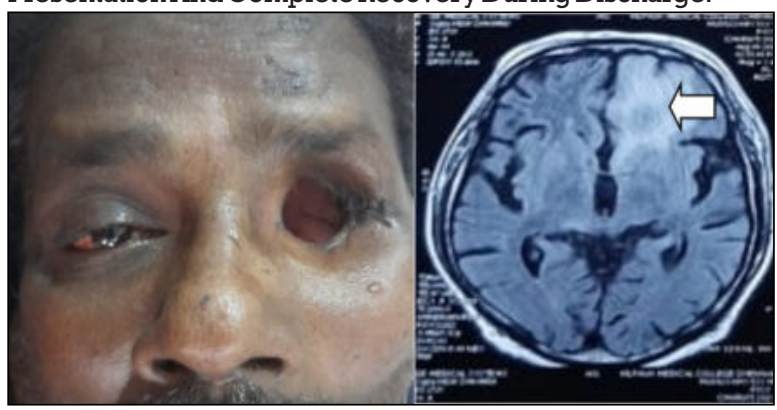

Fig-12: Picture Showing Anophthalmic Socket Post Orbital Exenteration Done For Stage-3c Patient \& MRI Brain Showing Intra Parenchymal Abcess In Left Basi Frontal Region

All patients had IDDM in uncontrolled status with one in diabetic ketoacidosis state. In three patients, hyperglycemia was uncontrollable despite treatment with insulin therapy. All patients had undergone a course of high dose corticosteroid therapy during hospitalisation for covid infection.

\section{DISCUSSION}

Mucor is a rare, opportunistic fungal infection causing an angioinvasive disease leading to aggressive necrosis and infarction of involved tissues and dissemination of infection.In India, there is an acute increase in the incidence of fungal infections, predominantly rhino-orbital mucormycosis in post covid patients. In normal individuals, mononuclear and polymorphonuclear phagocytes generate oxidative metabolites and defensins which kills mucorales. However, in covid patients, there is a profound lymphopenia with increase in neutrophil and monocyte counts leading to an imbalance between these cells making the patient more susceptible to systemic fungal infections.A study by Song $\mathbf{G}^{(11)}$ et al have concluded that impairment of $\mathrm{T}$ cell immunity with immunocompromised state predisposes to mucormycosis.

In our study, prevalence of mucormycosis was more common in males (64\%) with age preponderance between $51-60$ years $(28 \%)$. The time interval between post COVID-19 infection and Rhino orbital mucormycosis presentation was 7-14 days in majority of our cases.In our study, left eye was commonly involved in 14 patients (56\%) and right eye was involved in 3 patients (12\%). Bilateral presentation was seen in 8 patients (32\%).

In our study, 23 patients $(92 \%)$ had diabetes mellitus. Diabetes alters patient's immunological capability by inhibiting host phagocytosis and influx of polymorphonuclear leukocytes. Elevated blood glucose levels, serum ferritin levels and acidosis results in increase in availability of serum free iron which helps in the growth of fungus ${ }^{(12)}$. In covid patients, there is a transient hyperglycemia with an immunocompromised status making them prone to Rhino-orbital mucormycosis.

Mucormycosis occurs in varied clinical presentations. Among them Rhino -orbital-cerebral mucormycosis is the commonest form. The infection spreads up from nasal mucosa to sinuses, palate and orbit and finally to brain causing fatal complications. Jiang et $\mathrm{al}^{(13)}$., states that among orbital presentation, Orbital apex syndrome is rare and presents as retro-orbital pain, ptosis, complete ophthalmoplegia and visual loss. The orbitalpresentation is due to vascular tropism of the mucor fungus causing thrombosis of artery in orbital wall with thrombosis of central retinal artery. In our study, 7 patients presented as orbital apex syndrome.

Covid 19 is also a pro-coagulable state with increased incidence of thrombotic events. This also makes angioinvasive mucor more prone in post covid patients. CRAO is a rare manifestation of ROCM with an incidence of $16-20 \%$. It is due to direct infiltration of central retinal artery by angioinvasive mucor from the orbit. Bhansali et $\mathrm{al}^{(14)}$. and Yohai et $\mathrm{al}^{(7)}$. in their studies reported visual loss in mucormycosis due to CRAO and cavernous sinus thrombosis. Similarly in our study, visual loss was seen in five patients, out of which four were due to CRAO and one was due to cavernous sinus thrombosis.

Fundus examination plays a key role in diagnosing orbital apex syndrome which portraits venous congestion, optic disc edema and central retinal artery thrombosis. Early diagnosis and treatment has good prognosis in these patients. Otherwise intracranial involvement occurs via invasion of superior orbital fissure, ophthalmic vessels, cribriform plate, optic nerve and carotid artery. In our study most cases recovered with aggressive antifungal therapy, one patient was subjected to orbital exenteration to prevent fatal complications. However, one patient presented unique with initial intracranial involvement of cavernous sinus thrombosis with late onset of CRAO after 15 days.

In our study, the most common presenting symptom was nasal discharge with sinus and orbital pain. Though eschar was not present in all cases, MRI played a vital role in diagnosing and delineating the anatomical extent of lesion and its complications. This helps in detecting early vascular and intracranial involvement. Awal et $\mathrm{al}^{(15)}$,,describes imaging findings as thickening and opacification of paranasal sinuses, hypertrophy of nasal turbinates with secretions. In orbit, lesions appear hypointense on Tl weighted images and variable to hyperintense on $\mathrm{T} 2$ weighted images along with restricted diffusion on DWl. These can be seen within cavernous sinus, infratemporal fossa and skull base also. Similarly in our study, all patients presented with sinus involvement and 12 patients had orbital lesions. One patient presented with cavernous sinus thrombosis and infarction in internal capsule. Another patient presented with intracerebral abscess formation.

In covid patients, the use of steroids reduces the need of invasive ventilation in hypoxic patients and duration of hospital stay by promoting recovery and decreasing mortality. Steroids produces neutrophilic leukocytosis and 
lymphopenia with inhibition of migration of leukocytes to site of inflammation by inhibitory effects of cytokines.A study by Mehta et $\mathrm{al}^{(16)}$ repoted increased incidence of rhino orbital mucormycosis in post covid patients who received prolonged steroid therapy.In our study, all patients (100\%) had a course of prolonged high dose steroid therapy.

In our study, only 8 patients showed culture positive for mucormycosis. However, all patients showed positive mycelial filaments on histopathological staining with haematoxylin and eosin staining.The high number of culture negative findings but microscopically positive findings can be explained by fragile non septated growth of these fungi makes them easily mechanically damageable during sample manipulation.

Successful treatment of mucormycosis can be achieved with early diagnosis and aggressive therapy. This includes complete and thorough surgical debridement of infected tissues present in any region. In our study, all patients underwent FESS and complete debridement of infarcted tissues. One patient underwent orbital exenteration. No patient died due to fatal complications. This is similar to the study by Tedder et $\mathrm{al}^{(17)}$, who reported mortality rate was low $(11 \%)$ in patients who underwent surgery than without surgery $(60 \%)$.

Systemic Amphotericin-B administered intravenously at a dose of $1.0-1.5 \mathrm{mg} / \mathrm{kg}$ daily for 14 days with careful monitoring of renal parameters is associated with a good survival rate of $72 \%$. In our study all patients received systemic Amphotericin-B. In 12 patients with orbital extension, Retrobulbar injection of liposomal amphotericin was given to prevent its further spread Safi $\mathrm{M}$ et $\mathrm{al}^{(18)}$, described a good recovery rate following retrobulbar injection in their study.

Recent studies show good role of posoconazole, caspofungin, fluconazole in therapy. Hyperbaric oxygen therapy is also believed to improve neutrophilic killing by higher oxygen delivery.

In our study,patients who had uncontrolled diabetes mellitus despite insulin therapy, who were on prolonged steroid therapy, who had medial orbital wall involvement in MRI and who presented with central retinal artery occlusion as initial manifestation had a progression of disease. This makes more evident that these are important risk factors associated with progression of mucormycosis.

\section{Limitations OfThe Study}

As the patients were treated in different hospitals for covid pneumonia, blood parameters like serum ferritin, D-dimer levels, CRP values could not be collected properly. Hence their role as a risk factor for mucormycosis could not be analysed.

Due to late presentation of certain cases, exact time of onset of disease could not be determined. Incubation period of the disease per se cannot be concluded.

\section{CONCLUSION}

Mucormycosis is a severe, emergent and fatal infection requiring multidisciplinary approach in diagnosis and management. It is a disease with various presentations and atypical manifestations.The diagnosis relies on histological and mycological examination.

The most important risk factors for progression of mucormycosis are uncontrolled diabetes mellitus, prolonged steroid therapy, medial orbital wall involvement and artery thrombosis. All these risk factors needsto be closely monitored in convalescent phase for onset of mucormycosis and if infection occurs, for the progression of disease.
Earlier diagnosis and management of the patientshelps toprevent progression of disease and thereby visual loss and cosmetic disfiguration. Adequate and timely treatment of all patients with Retrobulbar Amphotericin and systemic posoconazole together with surgical debridement are important for successful eradication of infection and patient survival.

Due to changing trends in the covid pandemic it is an absolute necessary for all ophthalmologists to be aware of varying clinical presentations of rhino-orbital mucormycosis and its complications.

\section{REFERENCES:}

1. Ferguson BJ. Mucormycosis of the nose and paranasal sinuses. Otolaryngol Clin North Am. 2000;33(2):349-365.

2. Roden MM, Zaoutis TE, Buchanan WL, et al. Epidemiology and outcome of zygomycosis: a review of 929 reported cases. Clin Infect Dis. 2005;41(5):634653

3. Skiada A, Pavleas I, Drogari-Apiranthitou M. Epidemiology and diagnosis of mucormycosis: an update.J Fungi (Basel).2020;6(4):E265.

4. Chander J, Kaur M, Singla N, et al. Mucormycosis: battle with the deadly enemy over a five-year period in india.JoF.2018;4(2):46.

5. Bayram N, Ozsaygill C, Sav H, et al. Susceptibility of severe COVID-19 patients to rhino-orbital mucormycosis fungal infection in different clinical manifestations.Jpn J Ophthalmol. Published online May 31, 2021:1-11.

6. Lv Z, Cheng S, Le J, Huang J, Feng L, Zhang B, et al. Clinical characteristics and co-infections of 354 hospitalized patients with COVID-19 in Wuhan, China: a retrospective cohort study.Microbes Infect. 2020;22:195-9.

7. Yohai RA, Bullock JD, Aziz AA, Markert RJ. Survival factors in rhino-orbitalcerebral mucormycosis. SurvOphthalmol. 1994;39(1):3-22.

8. Schwartz JN, Donnelly EH, Klintworth GK. Ocular and orbital phycomycosis. SurvOphthalmol. 1977;22(1):3-28.

9. Press GA, Weindling SM, Hesselink JR, Ochi JW, Harris JP. Rhinocerebralmucormycosis: MR manifestations. J Comput Assist Tomogr. 1988;12(5):744-749.

10. McLean FM, Ginsberg LE, Stanton CA. Perineural spread of rhinocerebralmucormycosis. AJNR Am J Neuroradiol1996;17:114-6

11. Song G, Liang G, Liu W. Fungal co-infections associated with Global COVID19 pandemic: a clinical and diagnostic perspective from China. Mycopathologia.2020;185:599-606.

12. Chakrabarti A, Das A, Mandal J, et al.The rising trend of invasive zygomycosis in patients with uncontrolled diabetes mellitus. Med Mycol. 2006;44(4):335342.

13. Jiang N, Zhao G, Yang S, et al. A retrospective analysis of eleven cases of invasive rhino-orbito-cerebral mucormycosis presented with orbital apex syndrome initially2016;16:10.

14. Bhansali A, Bhadada S, Sharma A, Suresh V, Gupta A, Singh P, et al. Presentation and outcome of rhino-orbital-cerebral Mucormycosis in patients with diabetes. Postgrad Med J 2004;80:670-4

15. Awal SS, Biswas SS, Awal SK. Rhino-orbital mucormycosis in COVID-19 patients-a new threat? Egyptian Journal of Radiology and Nuclear Medicine. $2021 ; 52(1): 152$.

16. Mehta S, Pandey A. Rhino-Orbital Mucormycosis AssociatedWith COVID-19. Cureus. 2020;12(9):10726

17. Tedder M, Spratt JA, Anstadt MP, Hegde SS, Tedder SD, Lowe JE. Pulmonary mucormycosis: results of medical and surgical therapy. Ann Thorac Surg. 1994;57(4):1044-50.

18. Safi M, Ang MJ, Patel P, Silkiss RZ. Rhino-orbital-cerebral mucormycosis (ROCM) and associated cerebritis treated with adjuvant retrobulbar amphotericin B.Am J Ophthalmol Case Rep.2020;19:100771. 